\title{
The Elusive Renal Cell Carcinoma: Reversal Imaging of Arterial Phase to Improve Acuity
}

\author{
Erich K. Lang, Karl Zhang, Quan Nguyen, Daniel Thorner, Ernest Rudman \\ Department Radiology and Urology, SUNY Downstate Medical School, Brooklyn, New York, NY, USA
}

On routine physical examination the patient's physician noted microscopic hematuria The finding was reconfirmed by 2 Dipsticks over an interval of 4 months. The patient was a known diabetic, controlled by diet. Otherwise the patient was asymptomatic, without significant past medical history at the time of this work-up, the 47 year old Caucasian male appeared to be in good general health. Laboratory data showed $\mathrm{Hb}$ of $15.1 \mathrm{gm} / \mathrm{dL}$, HCT $45 \%$, RBC 4.8 million/uL, WBC 6200, Neu $62 \%$, BUN $18 \mathrm{mg} / \mathrm{dL}$, Creatinine $1.1 \mathrm{mg} / \mathrm{dl}$, GRF $94 \mathrm{~mL} / \mathrm{min}, \mathrm{A} / \mathrm{G}$ ratio 1.4, Glu $128 \mathrm{mg} / \mathrm{dl}$, K 4.2 $\mathrm{mmoL} / \mathrm{L}, \mathrm{Na} 145 \mathrm{MMOL} / \mathrm{L}$ Cl $108 \mathrm{mmoL} / \mathrm{L}$ Urine analysis, spec grav 1018, 3-5 RBC/hpf, no WBC or bacteria on hpf, no casts, urine culture negative $x$ 2. A KUB (Flat plate of abdomen) showed no opaque calculi nor other abnormalities. Cystoscopy and blue light cystoscopy revealed no abnormalities.

An enhanced 4 phase MDCT was performed The pre-enhancement phase was entirely unremarkable; no parenchymal lesions were detected. Following administration of $100 \mathrm{ml}$ nonionic contrast medium at a flow rate of $5 \mathrm{~mL} / \mathrm{sec}$, the 12 second delayed arterial phase $\mathrm{Ct}$ demonstrated a relatively poorly enhancing $1.6 \mathrm{~cm}$ mass at the cortico-medullary junction (Figure-1), the lesion is much better shown on reversal image. Both the parenchymal phase CT( 50 second delay) and the excretory phase CT (4 minutes delay Figure-2) demonstrate a non-enhancing $16 \mathrm{~mm}$ mass at the cortico-medullary junction (Figure-3).

In the light of a clinical history of diabetes and microscopic hematuria, the non-enhancing hypovascular mass seen on parenchymal and ex- cretory phase CTs in the medulla might have been written off as Medullary Necrosis (With characteristic CT findings of a negative pre-enhancement phase CT, but a non-enhancing lesion shown on parenchymal and excretory phase; an early avascular necrosis) (1). However, the reversal image of the arterial phase CT clearly shows an enhancing lesion, though somewhat hypovascular for a RCC. The hypo-density on parenchymal and excretory phase CT reflects the characteristic "wash-out" phenomenon of RCCs in these phases. The tumor having no tubules is less dense then adjacent normal parenchyma The correct diagnosis was made, and a laparoscopic resection carried out.

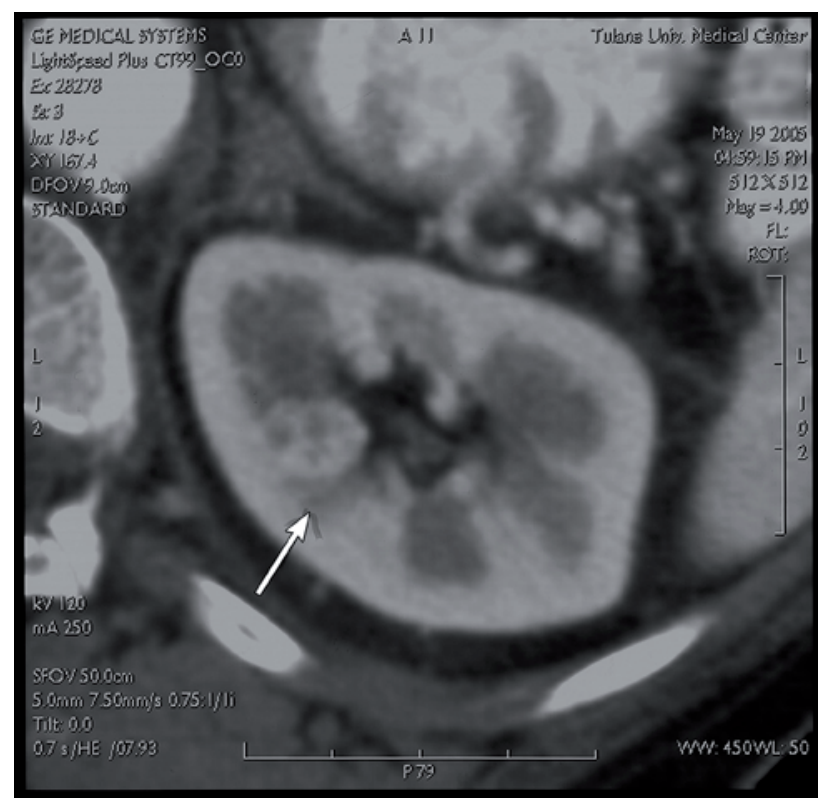

Figure 1 - Massive gas in the bladder, dissecting in the submucosal layer and extending into the space of Retzius. 


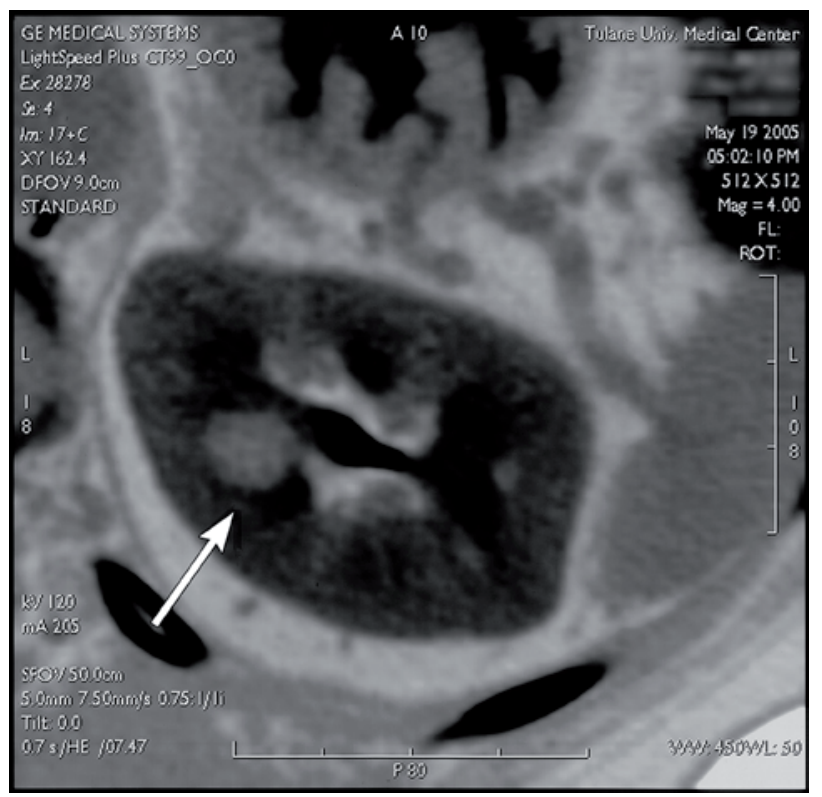

Figure 2 - lateral extension of the gas-dissection in the pre-vesical space.

\section{REFERENCES}

1. Lang EK, Macchia RJ, Thomas R, Davis R, RuizDeya G, Watson RA, et al.: Multiphasic helical CT diagnosis of early medullary and papillary necrosis.

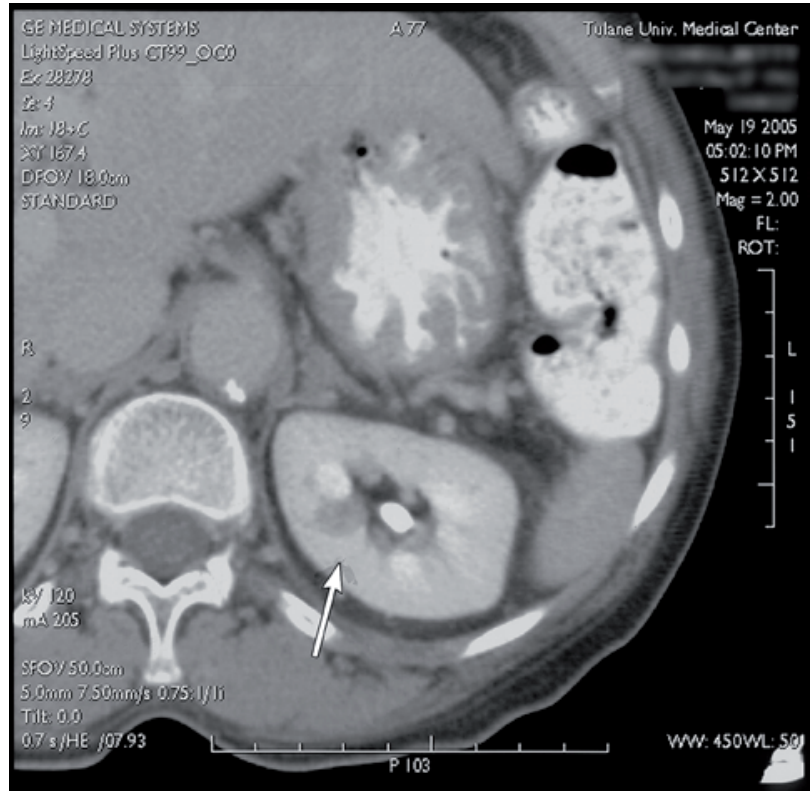

Figure 3 - gas dissecting into the ureter.
J Endourol. 2004; 18: 49-56.

2. Israel GM, Bosniak MA: How I do it: evaluating renal masses. Radiology. 2005; 236: 441-50.

\author{
Correspondence address: \\ Dr. Erich K. Lang \\ Departments of Urology and Radiology \\ SUNY, Downstate Health Science Center \\ 455 Lenox Road \\ Brooklyn, NY, 11203, USA \\ E-mail: erich.lang@downstate.edu
}

